\title{
INEQUALITIES FOR ZERO-BALANCED HYPERGEOMETRIC FUNCTIONS
}

\author{
G. D. ANDERSON, R. W. BARNARD, K. C. RICHARDS, \\ M. K. VAMANAMURTHY, AND M. VUORINEN
}

ABSTRACT. The authors study certain monotoneity and convexity properties of the Gaussian hypergeometric function and those of the Euler gamma function.

\section{INTRODUCTION}

The Gaussian hypergeometric function is defined by

$$
F(a, b ; c ; x)={ }_{2} F_{1}(a, b ; c ; x)=\sum_{n=0}^{\infty} \frac{(a, n)(b, n)}{(c, n)} \frac{x^{n}}{n !},
$$

for $x \in(-1,1)$, where $(a, n)$ denotes the shifted factorial function $(a, n)=$ $a(a+1) \cdots(a+n-1), n=1,2, \ldots$, and $(a, 0)=1$ for $a \neq 0$. The sum is well defined at least when $(c, n) \neq 0$, i.e., when $c \neq 0,-1,-2, \ldots$ This function has found frequent applications in various fields of the mathematical and natural sciences [Ask2]. Many elementary transcendental functions are special cases or limiting cases of $F(a, b ; c ; x)$; for an extensive list see [AS, pp. 556-566], [PBM, pp. 430-615]. Two important special cases are the complete elliptic integrals

$$
\mathscr{K}(x)=\frac{\pi}{2} F\left(\frac{1}{2}, \frac{1}{2} ; 1 ; x^{2}\right), \quad \mathscr{E}(x)=\frac{\pi}{2} F\left(-\frac{1}{2}, \frac{1}{2} ; 1 ; x^{2}\right) .
$$

As a function of its parameters $a, b, c$ the function $F(a, b ; c ; x)$ is smooth, and it is thus natural to expect that the properties of $\mathscr{K}(x)$ extend also to $F\left(a, b ; c ; x^{2}\right)$ for $(a, b, c)$ close to $\left(\frac{1}{2}, \frac{1}{2}, 1\right)$. Recall that $F(a, b ; c ; x)$ is called zero-balanced if $c=a+b$. We obtain several results for the zerobalanced $F(a, b ; a+b ; x), a, b>0$, extending well-known properties of $\mathscr{K}(x)$.

1.3. Theorem. (1) For $a, b \in(0, \infty)$ the function

$$
f(x) \equiv \frac{1-F(a, b ; a+b ; x)}{\log (1-x)}
$$

Received by the editors September 17,1993; originally communicated to the Proceedings of the $A M S$ by Hal L. Smith.

1991 Mathematics Subject Classification. Primary 33C05, 33B15; Secondary 26D07.

Key words and phrases. Hypergeometric function, gamma function, elliptic integral. 
is strictly increasing from $(0,1)$ onto $(a b /(a+b), 1 / B)$, where $B=B(a, b)$ is the Euler beta function.

(2) For $a, b \in(0, \infty)$ the function

$$
g(x) \equiv B F(a, b ; a+b ; x)+\log (1-x)
$$

is strictly decreasing from $(0,1)$ onto $(R, B)$, where

$$
R=-\Psi(a)-\Psi(b)-2 \gamma \text {. }
$$

Here $\Psi(a)=\Gamma^{\prime}(a) / \Gamma(a)$, and $\gamma$ is the Euler-Mascheroni constant.

1.4. Theorem. For $a, b \in(0, \infty)$, let

$$
f(x)=x F(a, b ; a+b ; x) / \log (1 /(1-x))
$$

on $(0,1)$ and let $B, R$ be as in Theorem 1.3 .

(1) If $a, b \in(0,1)$, then $f$ is decreasing with range $(1 / B, 1)$.

(2) If $a, b \in(1, \infty)$, then $f$ is increasing with range $(1,1 / B)$.

(3) If $a=b=1$, then $f(x)=1$ for all $x \in(0,1)$.

(4) If $a, b \in(0,1)$ the function $g_{1}(x) \equiv B F(a, b ; a+b ; x)+(1 / x) \log (1-x)$ is increasing from $(0,1)$ onto $(B-1, R)$.

(5) If $a, b \in(1, \infty)$, then $g_{1}$ is decreasing from $(0,1)$ onto $(R, B-1)$.

Here Theorem 1.3(1) generalizes the fact that $((2 / \pi) \mathscr{K}(x)-1) / \log \left(1 / x^{\prime}\right)$ is increasing from $(0,1)$ onto $(1 / 2,2 / \pi) ; 1.3(2)$ generalizes the well-known fact that $\mathscr{K}(x)+\log x^{\prime}$ is decreasing from $(0,1)$ onto $(\log 4, \pi / 2)$ (cf. [AVV1, Theorem 2.2(2)]); and 1.4(1) generalizes the result that $x^{2} \mathscr{K}(x) / \log \left(1 / x^{\prime}\right)$ is decreasing from $(0,1)$ onto $(1, \pi)$ [AVV3, Theorem 2(19)].

The asymptotic relation

$$
F(a, b ; a+b ; x) \sim-\frac{1}{B(a, b)} \log (1-x)
$$

as $x \rightarrow 1$ is due to Gauss, and its refined form

$$
B(a, b) F(a, b ; a+b ; x)+\log (1-x)=R+O((1-x) \log (1-x))
$$

as $x \rightarrow 1$, with $R=-\Psi(a)-\Psi(b)-2 \gamma$, is due to S. Ramanujan [Ev, p. 553], [Be, p. 71]. Theorems 1.3 and 1.4 are refinements of these classical relations. Ramanujan also gave extensions of (1.6) to the generalized hypergeometric function ${ }_{p} F_{q}$ for certain values of $p$ and $q$ [Ev, pp. 553-558], [Be, pp. 12, 71]. Formulas (1.5) and (1.6) follow from the identity in [AS, 15.3.10], which has been generalized recently in [B, p. 152].

1.7. Theorem. For each $a, b \in(0, \infty)$ the function

$$
f(x) \equiv(1-x)^{1 / 4} F(a, b ; a+b ; x)
$$

is a strictly decreasing automorphism of $(0,1)$ if and only if $4 a b \leq a+b$.

While studying relationships between the arithmetic-geometric mean and some other means, J. and P. Borwein [BB2, (2.9)] recently proved that

$$
F\left(\frac{1}{2}, \frac{1}{2} ; 1 ; 1-x^{c}\right)<F\left(\frac{1}{2}-\delta, \frac{1}{2}+\delta ; 1 ; 1-x^{d}\right)
$$

for all $x \in(0,1)$, with $c=2, d=3, \delta=\frac{1}{6}$.

We obtain the following generalization. 
1.9. Theorem. For $c, d \in(0, \infty), 4 c<\pi d$, inequality (1.8) holds for all $x \in(0,1)$ and for all $\delta \in\left(0, \delta_{o}\right)$, where $\delta_{o}=((d \pi-4 c) /(4 \pi d))^{1 / 2}$.

We conclude the paper by proving some inequalities for the gamma function. Our notation is mostly standard. For $x \in[0,1]$, we often denote $x^{\prime}=\sqrt{1-x^{2}}$.

\section{Proofs}

For many important properties of the functions $F(a, b ; c ; x), \mathscr{K}(x)$, and $\mathscr{E}(x)$ see [AS, pp. 556-566, 589-626], [C, Chapters 2, 9], [PBM, pp. 430-615]. Some algorithms for computing these functions are given in [Ba, pp. 345-372, 416-424], [M, Chapter 7], [PNB, p. 249], and [PT, pp. 404-426]. Various aspects of the theory of special functions are surveyed in [Ask1], [Ask2], [AVV3], [LO, 5.3, 5.5].

We shall give here a proof of the Gauss asymptotic formula (1.5), since we have been unable to find a proof in the recent literature or in the standard texts. Various generalizations of (1.5) appear in [Ev, pp. 553-558], [B, p. 152].

2.1. Lemma. (1) For $a, b \in(0, \infty)$, the sequence

$$
f(n) \equiv \frac{(a, n)(b, n)}{(a+b, n)(n-1) !}
$$

is increasing to the limit $1 / B(a, b)$, as $n \rightarrow \infty$.

(2) For $a, b \in(0,1)$, the sequence

$$
g(n) \equiv \frac{(a, n)(b, n)(n+1)}{(a+b, n) n !}
$$

is decreasing to the limit $1 / B(a, b)$, as $n \rightarrow \infty$.

(3) For $a, b \in(1, \infty)$, the sequence $g(n)$ is increasing to the limit $1 / B(a, b)$, as $n \rightarrow \infty$.

In particular, for each positive integer $n$,

$$
\begin{aligned}
& \frac{a b}{a+b} \leq f(n)<\frac{1}{B(a, b)} \text { for all } a, b \in(0, \infty), \\
& \frac{1}{B(a, b)}<g(n) \leq \frac{2 a b}{a+b} \quad \text { for all } a, b \in(0,1), \\
& \frac{2 a b}{a+b} \leq g(n)<\frac{1}{B(a, b)} \quad \text { for all } a, b \in(1, \infty),
\end{aligned}
$$

where the weak inequalities reduce to equality if and only if $n=1$.

Proof. Part (1) is proved in [AVV3, Theorem 6(4)]. For (2) and (3),

$$
\frac{g(n+1)}{g(n)}=\frac{(a+n)(b+n)(n+2)}{(a+b+n)(n+1)^{2}}<1
$$

iff $w_{1} \equiv((a+b+n) n+a b)(n+2)<w_{2} \equiv(a+b+n)(n+1)^{2}$, which is true since $w_{2}-w_{1}=(a+b-2 a b)+n(1-a b)$ is positive if $a, b \in(0,1)$, and negative if $a, b \in(1, \infty)$. The limiting values follow form Stirling's formula [C, p. 24], [Mi, p. 184]. 
2.2. Remark. The following inequalities due to Wallis appear in [Mi, p. 192]:

$$
\frac{1}{\sqrt{\pi\left(n+\frac{1}{2}\right)}}<\frac{\left(\frac{1}{2}, n\right)}{n !}<\frac{1}{\sqrt{\pi n}} .
$$

Since $B\left(\frac{1}{2}, \frac{1}{2}\right)=\pi$, the result in Lemma 2.1(4) generalizes the second Wallis inequality here.

2.3. Lemma (Gauss). For $a, b \in(0, \infty)$, the relation (1.5) holds.

Proof. Let $B=B(a, b)$. From Lemma 2.1, we have

$$
F(a, b ; a+b ; x)=\sum_{n=0}^{\infty} \frac{(a, n)(b, n)}{(a+b, n) n !} x^{n} \leq 1+\sum_{n=1}^{\infty} \frac{1}{B} \frac{x^{n}}{n}=1-\frac{1}{B} \log (1-x) .
$$

Hence

$$
\limsup _{x \rightarrow 1} \frac{F(a, b ; a+b ; x)}{\log (1 /(1-x))} \leq \frac{1}{B} .
$$

Next, fix $\varepsilon \in(0,1 / B)$. Then by Lemma 2.1 there exists a positive integer $n_{o}$ such that $(a, n)(b, n) /((a+b, n)(n-1) !)>(1 / B)-\varepsilon$, for all $n \geq n_{o}$. Hence, $F(a, b ; a+b ; x) \geq F_{n_{o}}+\left(\frac{1}{B}-\varepsilon\right) \sum_{n=n_{o}+1}^{\infty} \frac{x^{n}}{n}=F_{n_{o}}-\left(\frac{1}{B}-\varepsilon\right)\left(\log (1-x)+S_{n_{o}}\right)$, where

$$
F_{n_{o}}=\sum_{n=1}^{n_{o}} \frac{(a, n)(b, n)}{(a+b, n) n !} x^{n}, \quad S_{n_{o}}=\sum_{n=1}^{n_{o}} \frac{x^{n}}{n} .
$$

Dividing by $\log (1 /(1-x))$ and letting $x \rightarrow 1$, since $\varepsilon \in(0,1 / B)$ is arbitrary, we get

$$
\liminf _{x \rightarrow 1} \frac{F(a, b ; a+b ; x)}{\log (1 /(1-x))} \geq \frac{1}{B},
$$

so that the limit is $1 / B$.

2.4. Proof of Theorem 1.3. (1) The limiting value as $x \rightarrow 0$ follows from series expansions, while the one as $x \rightarrow 1$ follows from Lemma 2.3. Next, let $g(x)=F(a, b ; a+b ; x)-1$ and $h(x)=\log (1 /(1-x))$. Then $g(0)=h(0)=0$, and by the monotone l'Hôpital rule [AVV4, Lemma 2.2] it is enough to show that $g^{\prime}(x) / h^{\prime}(x)$ is strictly increasing. Now [WW, p. 281]

$$
\begin{aligned}
& \frac{g^{\prime}(x)}{h^{\prime}(x)}=\frac{a b}{a+b}(1-x) F(a+1, b+1 ; a+b+1 ; x) \\
&=\frac{a b}{a+b}\left(1+\sum_{n=0}^{\infty}\left(\frac{(a+1, n+1)(b+1, n+1)}{(a+b+1, n+1)(n+1) !}\right.\right. \\
&\left.\left.-\frac{(a+1, n)(b+1, n)}{(a+b+1, n) n !}\right) x^{n+1}\right) .
\end{aligned}
$$

The coefficient of $x^{n+1}$ here is positive if and only if $(a+1+n)(b+1+n)>$ $(a+b+n+1)(n+1)$, which holds if and only if $a b+(a+b)(n+1)+(n+1)^{2}>$ $(a+b)(n+1)+(n+1)^{2}$, which is true. 
(2) The limiting values are clear from (1.6). Next, by series expansion,

$$
g(x)-B=\sum_{n=1}^{\infty}\left(B \frac{(a, n)(b, n)}{(a+b, n)(n-1) !}-1\right) \frac{x^{n}}{n},
$$

so that all coefficients are negative by Lemma 2.1(4).

2.5. Proof of Theorem 1.4. For (1), let $g(x)=x F(a, b ; c ; x), c=a+b$, and $h(x)=\log (1 /(1-x))$. Then $g(0)=h(0)=0$ and

$$
\begin{aligned}
\frac{g^{\prime}(x)}{h^{\prime}(x)}= & (1-x)\left(F(a, b ; c ; x)+\frac{a b x}{c} F(a+1, b+1 ; c+1 ; x)\right) \\
= & (1-x)\left(\sum_{n=0}^{\infty} \frac{(a, n)(b, n)}{(c, n)} \frac{x^{n}}{n !}+\frac{a b}{c} \sum_{n=0}^{\infty} \frac{(a+1, n)(b+1, n)}{(c+1, n)} \frac{x^{n+1}}{n !}\right) \\
= & (1-x)\left(\sum_{n=0}^{\infty} \frac{(a, n)(b, n)}{(c, n)} \frac{x^{n}}{n !}+\sum_{n=0}^{\infty} \frac{(a, n+1)(b, n+1)}{(c, n+1)} \frac{x^{n+1}}{n !}\right) \\
= & \sum_{n=0}^{\infty} \frac{(a, n)(b, n)}{(c, n)} \frac{x^{n}}{n !}+\sum_{n=0}^{\infty}\left(\frac{(a, n+1)(b, n+1)}{(c, n+1)}-\frac{(a, n)(b, n)}{(c, n)}\right) \frac{x^{n+1}}{n !} \\
& -\sum_{n=0}^{\infty} \frac{(a, n+1)(b, n+1)}{(c, n+1)} \frac{x^{n+2}}{n !} .
\end{aligned}
$$

Hence

$$
\begin{aligned}
\frac{g^{\prime}(x)}{h^{\prime}(x)}-1= & \left(\frac{2 a b}{c}-1\right) x+\sum_{n=0}^{\infty} \frac{(a, n+2)(b, n+2)}{(c, n+2)} \frac{x^{n+2}}{(n+2) !} \\
& +\sum_{n=0}^{\infty} \frac{(a, n+2)(b, n+2)}{(c, n+2)} \frac{x^{n+2}}{(n+1) !}-\sum_{n=0}^{\infty} \frac{(a, n+1)(b, n+1)}{(c, n+1)} \frac{x^{n+2}}{n !} \\
& -\sum_{n=0}^{\infty} \frac{(a, n+1)(b, n+1)}{(c, n+1)} \frac{x^{n+2}}{(n+1) !} \\
= & \left(\frac{2 a b}{c}-1\right) x+\sum_{n=0}^{\infty} \frac{(a, n+1)(b, n+1)}{(c, n+2)(n+2) !} \\
& \quad \times[(n+1)(a b-1)+(2 a b-a-b)] x^{n+2},
\end{aligned}
$$

in which all coefficients are negative. Thus $g^{\prime}(x) / h^{\prime}(x)$ is decreasing, hence so is $g(x) / h(x)$, by the monotone l'Hôpital rule [AVV4, Lemma 2.2], and (1) follows.

The proof for (2) is similar, except that all coefficients are positive.

Part (3) follows from (1.1) and the series for $\log (1 /(1-x))$.

(4) In the series expansion,

$$
g_{1}(x)-(B-1)=\sum_{n=1}^{\infty}\left(\frac{(a, n)(b, n)}{(a+b, n) n !} B-\frac{1}{n+1}\right) x^{n},
$$

all coefficients are positive by Lemma $2.1(5)$.

(5) The proof is similar to (4), except that all coefficients are negative by Lemma 2.1(6). The limiting values are clear by (1.6). 
2.6. Lemma. For $a, b \in(0, \infty), n=1,2,3, \ldots$,

$$
\sum_{k=0}^{n} \frac{(a, k)(b, k)}{(a+b, k) k !}>\frac{(a+1, n)(b+1, n)}{(a+b+1, n) n !}>\frac{(a, n)(b, n)}{(a+b, n) n !} .
$$

Proof. The first inequality follows by induction, and the second one by the factorial property of $(a, n)$.

2.7. Lemma. For $a, b \in(0, \infty)$ and $x \in(0,1)$ the Maclaurin series of the functions

$$
\frac{1}{1-x} F(a, b ; a+b ; x)-F(a+1, b+1 ; a+b+1 ; x)
$$

and

$$
F(a+1, b+1 ; a+b+1 ; x)-F(a, b ; a+b ; x)
$$

have constant term zero and all other coefficients strictly positive. In particular, these functions are increasing and convex on $(0,1)$ and

$$
F(a, b ; a+b ; x)<F(a+1, b+1 ; a+b+1 ; x)<\frac{1}{1-x} F(a, b ; a+b ; x)
$$

for all $x \in(0,1)$.

Proof. The results follow immediately from Lemma 2.6.

2.8. Proof of Theorem 1.7. The limiting value $f(0)=1$ is obvious, while $f(1-)=0$ follows from Theorem 1.3(1). Next, for each $x \in(0,1)$ we have

$$
4(1-x)^{-1 / 4} f^{\prime}(x)=\frac{4 a b}{a+b} F(a+1, b+1 ; a+b+1 ; x)-\frac{1}{1-x} F(a, b ; a+b ; x) \text {. }
$$

If $4 a b \leq a+b$, then Lemma 2.7 implies that $f^{\prime}(x)<0$. Conversely, if $f$ is decreasing, then $f^{\prime}(x) \leq 0$ for all $x \in(0,1)$, and letting $x$ tend to 0 we get $4 a b \leq a+b$.

The special case $a=b=\frac{1}{2}$ of Theorem 1.7 is well known. This special case follows, for instance, from the infinite product formulas for $x^{\prime} / \sqrt{x}$ and $(2 / \pi) \sqrt{x} \mathscr{K}(x)$ in terms of the Jacobi nome $q=\exp \left(-\pi \mathscr{K}^{\prime} / \mathscr{K}\right)[\mathrm{WW}, \mathrm{p} .488$, Exercise 10] or from [AVV1, Theorem 2.2(3)].

2.9. Remark. It is easy to show that $(\sqrt{a b}, n)^{2} \leq(a, n)(b, n) \leq$ $((a+b) / 2, n)^{2}$ for all positive $a, b$ and all $n=1,2, \ldots$, with equality if and only if $a=b$. Thus

$$
F(\sqrt{a b}, \sqrt{a b} ; c ; x) \leq F(a, b ; c ; x) \leq F((a+b) / 2,(a+b) / 2 ; c ; x)
$$

for $a, b, c>0, x \in[0,1)$, with equality if and only if $a=b$ or $x=0$. Furthermore, for $a, c>0$ and for $t \in(0, a)$ we see that $(a+t, n)(a-t, n)$ is a decreasing function of $t$ on $[0, a]$ for $n=1,2, \ldots$, so that

$$
F\left(a+t_{2}, a-t_{2} ; c ; x\right) \leq F\left(a+t_{1}, a-t_{1} ; c ; x\right)
$$

for $x \in(0,1), 0 \leq t_{1}<t_{2} \leq a$. The second assertion of Lemma 2.7 also follows from the fact that $(a, n)(b, n) /(a+b, n), a, b>0$, is a strictly increasing function of $a$. 


\section{The gamma function}

We next study some properties of the functions

$$
\Gamma(x)=\int_{0}^{\infty} t^{x-1} e^{-t} d t \text { and } \Psi(x)=\frac{d}{d x} \log \Gamma(x)
$$

when $x$ is real and positive.

3.1. Theorem. The function $f(x)=x(\log x-\Psi(x))$ is decreasing and convex from $(0, \infty)$ onto $\left(\frac{1}{2}, 1\right)$.

Proof. From [WW, p. 251, §12.32, Example] it follows that

$$
f(x)=\frac{1}{2}+2 x \int_{0}^{\infty} \frac{t d t}{\left(t^{2}+x^{2}\right)\left(e^{2 \pi t}-1\right)} .
$$

Hence,

$$
\begin{aligned}
f^{\prime}(x) & =2 \int_{0}^{\infty} \frac{t d t}{\left(t^{2}+x^{2}\right)\left(e^{2 \pi t}-1\right)}-4 x^{2} \int_{0}^{\infty} \frac{t d t}{\left(t^{2}+x^{2}\right)^{2}\left(e^{2 \pi t}-1\right)} \\
& =2 \int_{0}^{\infty} \frac{t\left(t^{2}-x^{2}\right) d t}{\left(t^{2}+x^{2}\right)^{2}\left(e^{2 \pi t}-1\right)} \\
& =2 \int_{0}^{x} \frac{t}{e^{2 \pi t}-1} \frac{\left(t^{2}-x^{2}\right) d t}{\left(t^{2}+x^{2}\right)^{2}}+2 \int_{x}^{\infty} \frac{t}{e^{2 \pi t}-1} \frac{\left(t^{2}-x^{2}\right) d t}{\left(t^{2}+x^{2}\right)^{2}}
\end{aligned}
$$

Since $t /\left(e^{2 \pi t}-1\right)$ is decreasing on $(0, \infty)$, we get

$$
f^{\prime}(x)<\frac{2 x}{e^{2 \pi x}-1} \int_{0}^{\infty} \frac{\left(t^{2}-x^{2}\right) d t}{\left(t^{2}+x^{2}\right)^{2}} .
$$

Substituting $t=x \tan u$, we get

$$
\int_{0}^{\infty} \frac{\left(t^{2}-x^{2}\right) d t}{\left(t^{2}+x^{2}\right)^{2}}=\frac{1}{x} \int_{0}^{\pi / 2}\left(\sin ^{2} u-\cos ^{2} u\right) d u=0
$$

Thus we have shown that $f(x)$ is strictly decreasing, so that $f(0+)$ exists. To obtain this limit we observe first that $t /\left(e^{2 \pi t}-1\right)<1 /(2 \pi)$ implies that

$$
\lim _{x \rightarrow 0+} 2 x \int_{0}^{\infty} \frac{t d t}{\left(t^{2}+x^{2}\right)\left(e^{2 \pi t}-1\right)} \leq \frac{1}{2}
$$

Next, fix $\varepsilon \in(0,1 /(2 \pi))$. Since $\lim _{t \rightarrow 0+} t /\left(e^{2 \pi t}-1\right)=1 /(2 \pi)$, there exists $\delta>0$ such that $t /\left(e^{2 \pi t}-1\right)>1 /(2 \pi)-\varepsilon$ for all $t \in(0, \delta)$. Thus

$$
2 x \int_{0}^{\infty} \frac{t d t}{\left(t^{2}+x^{2}\right)\left(e^{2 \pi t}-1\right)}>2 x\left(\frac{1}{2 \pi}-\varepsilon\right) \int_{0}^{\delta} \frac{d t}{t^{2}+x^{2}}=2\left(\frac{1}{2 \pi}-\varepsilon\right) \arctan \frac{\delta}{x} .
$$

Now letting first $x$, then $\varepsilon$, tend to zero gives

$$
\lim _{x \rightarrow 0+} 2 x \int_{0}^{\infty} \frac{t d t}{\left(t^{2}+x^{2}\right)\left(e^{2 \pi t}-1\right)} \geq \frac{1}{2} .
$$

For $0 \leq a<b \leq \infty$ denote

$$
I(a, b)=\int_{a}^{b} \frac{t d t}{\left(t^{2}+x^{2}\right)\left(e^{2 \pi t}-1\right)} .
$$


Then, since $t /\left(e^{2 \pi t}-1\right) \leq 1 /(2 \pi)$,

$$
2 x I(0,1) \leq \frac{1}{\pi} \arctan \frac{1}{x}
$$

while

$$
\begin{aligned}
I(1, \infty) & \leq \frac{1}{1+x^{2}} \int_{1}^{\infty} \frac{t d t}{e^{2 \pi t}-1}=\frac{1}{1+x^{2}} \int_{1}^{\infty} \frac{t e^{-\pi t} d t}{2 \sinh (\pi t)} \\
& \leq \frac{1}{2 \pi\left(1+x^{2}\right)} \int_{1}^{\infty} e^{-\pi t} d t=\frac{1}{2 \pi^{2}\left(1+x^{2}\right)}
\end{aligned}
$$

Hence $\lim _{x \rightarrow \infty} f(x)=\frac{1}{2}$.

To prove the convexity, let $J[g]$ denote the integral of a function $g$ from 0 to $\infty$. Then, as above, $f^{\prime}(x)=2 J[g(t) h(t, x)]$, where

$$
g(t)=t /\left(e^{2 \pi t}-1\right) \text { and } h(t, x)=\left(t^{2}-x^{2}\right) /\left(t^{2}+x^{2}\right)^{2} .
$$

Hence,

$$
f^{\prime \prime}(x)=4 x J[g(t) H(t, x)], \quad \text { where } H(t, x)=\frac{\partial h(t, x)}{\partial x}=\frac{x^{2}-3 t^{2}}{\left(t^{2}+x^{2}\right)^{3}} .
$$

Since $g(t)$ is decreasing, by splitting the integral on $(0, \infty)$ into the sum of an integral on $(0, x / \sqrt{3})$ and one on $(x / \sqrt{3}, \infty)$, we get

$$
f^{\prime \prime}(x)>4 x g(x / \sqrt{3}) J[H(t, x)] \text {. }
$$

Now substituting $t=x \tan u$, we see that $J[H(t, x)]=0$. Hence $f^{\prime \prime}(x)>0$ on $(0, \infty)$, so that $f$ is convex on $(0, \infty)$.

3.2. Theorem. (1) The function $f_{1}(x)=x^{1 / 2-x} e^{x} \Gamma(x)$ is decreasing and logconvex from $(0, \infty)$ onto $(\sqrt{2 \pi}, \infty)$.

(2) The function $f_{2}(x)=x^{1-x} e^{x} \Gamma(x)$ is increasing and log-concave from $(0, \infty)$ onto $(1, \infty)$.

Proof. (1) The limiting value at 0 follows from the relation $\Gamma(x+1)=x \Gamma(x)$, while the one at $\infty$ follows from Stirling's formula. Next,

$$
-x \frac{d}{d x} \log f_{1}(x)=f(x)-\frac{1}{2},
$$

where $f(x)$ is as in Theorem 3.1 , is clearly positive and decreasing.

(2) The limiting value at 0 follows from the relation $\Gamma(x+1)=x \Gamma(x)$, while the one at $\infty$ follows from Stirling's formula. Next,

$$
-x \frac{d}{d x} \log f_{2}(x)=1-f(x)>0,
$$

where $f(x)$ is as in Theorem 3.1. Moreover, by the monotone l'Hôpital rule [AVV4, §2], it follows that $\frac{d}{d x} \log f_{2}(x)$ is decreasing.

3.3. Remark. A result similar to Theorem 3.2 appears in [Lu, p. 17], whereas in [Mi, 3.6.55, p. 288] a version of Theorem 3.1 is given. In [G, p. 283] it is 
shown that $x \Psi(x)$ is convex for $x>0$. For some recent results on the gamma function see [A1].

\section{REFINEMENTS}

A problem of interest is to obtain upper estimates for the complete elliptic integral

$$
\frac{2}{\pi} \mathscr{K}\left(x^{\prime}\right)=F\left(\frac{1}{2}, \frac{1}{2} ; 1 ; 1-x^{2}\right)
$$

in terms of variants of the type

$$
F\left(\frac{1}{2}-\delta, \frac{1}{2}+\delta ; 1 ; 1-x^{3}\right)
$$

for $x \in(0,1)$ and $\delta \in\left(0, \frac{1}{2}\right)$. Observe initially that the inequality

$$
F\left(\frac{1}{2}, \frac{1}{2} ; 1 ; 1-x^{2}\right)<F\left(\frac{1}{2}, \frac{1}{2} ; 1 ; 1-x^{3}\right)
$$

for $x \in(0,1)$ follows immediately from the monotoneity of $F\left(\frac{1}{2}, \frac{1}{2} ; 1 ; x\right)$ as a function of $x$. We seek a refinement of (4.1).

J. Borwein and P. Borwein [BB2, (2.9)] have shown that

$$
F\left(\frac{1}{2}, \frac{1}{2} ; 1 ; 1-x^{2}\right)<F\left(\frac{1}{2}-\delta, \frac{1}{2}+\delta ; 1 ; 1-x^{3}\right)
$$

for $\delta=\frac{1}{6}$ and for all $x \in(0,1)$. It has been conjectured recently [AVV3, p. 79] that (4.2) holds for all $\delta \in\left(0, \frac{1}{6}\right)$ and for all $x \in(0,1)$. We next obtain a refinement of (4.2), which also proves the statement of Theorem 1.9.

4.3. Theorem. Let $x \in(0,1), c, d \in(0, \infty), 4 c<\pi d$. Then

$$
\begin{aligned}
F\left(\frac{1}{2}, \frac{1}{2} ; 1 ; 1-x^{c}\right) & <F\left(\frac{1}{2}-\delta_{0}, \frac{1}{2}+\delta_{0} ; 1 ; 1-x^{d}\right) \\
& <F\left(\frac{1}{2}-\delta, \frac{1}{2}+\delta ; 1 ; 1-x^{d}\right) \\
& <F\left(\frac{1}{2}, \frac{1}{2} ; 1 ; 1-x^{d}\right)
\end{aligned}
$$

for all $\delta \in\left(0, \delta_{0}\right)$, where $\delta_{0}=((d \pi-4 c) /(4 \pi d))^{1 / 2}$.

Proof. Let $f_{\delta}(x)$ be defined by

$$
f_{\delta}(x)=\frac{1-F\left(\frac{1}{2}-\delta, \frac{1}{2}+\delta ; 1 ; x\right)}{\log (1-x)} .
$$

It follows from Theorem 1.3 that, for all $x \in(0,1)$ and $\delta \in\left[0, \frac{1}{2}\right)$,

$$
\left(\frac{1}{2}-\delta\right)\left(\frac{1}{2}+\delta\right)<f_{\delta}(x)<\frac{1}{B\left(\frac{1}{2}-\delta, \frac{1}{2}+\delta\right)},
$$

where $B(a, b)$ is the Euler beta function. From the reflection formula $\Gamma(a) \Gamma(1-a)=\pi / \sin (a \pi)$ [AS, p. 256], it follows that

$$
\frac{1}{4}-\delta^{2}<f_{\delta}(x)<\frac{1}{\pi} \sin \left(\left(\frac{1}{2}-\delta\right) \pi\right)
$$


for all $x \in(0,1)$ and $\delta \in\left[0, \frac{1}{2}\right)$. From (4.9) with $\delta=0$ it follows that

$$
\frac{F\left(\frac{1}{2}, \frac{1}{2} ; 1 ; 1-x^{c}\right)-1}{\log \left(1 / x^{c}\right)}=f_{0}\left(1-x^{c}\right)<\frac{1}{\pi} \text {. }
$$

Again applying (4.9) with $\delta \in\left(0, \delta_{0}\right)$ yields

$$
\begin{aligned}
\frac{F\left(\frac{1}{2}-\delta, \frac{1}{2}+\delta ; 1 ; 1-x^{d}\right)-1}{\log \left(1 / x^{c}\right)} & =\frac{d}{c}\left[\frac{F\left(\frac{1}{2}-\delta, \frac{1}{2}+\delta ; 1 ; 1-x^{d}\right)-1}{\log \left(1 / x^{d}\right)}\right] \\
& =\frac{d}{c} f_{\delta}\left(1-x^{d}\right)>\frac{d}{c}\left(\frac{1}{4}-\delta^{2}\right) \\
& >\frac{d}{c}\left(\frac{1}{4}-\delta_{0}^{2}\right)=\frac{1}{\pi} .
\end{aligned}
$$

Therefore, for $0<\delta \leq \delta_{0}$ and $x \in(0,1)$,

$$
\begin{aligned}
F\left(\frac{1}{2}, \frac{1}{2} ; 1 ; 1-x^{c}\right) & =1+f_{0}\left(1-x^{c}\right) \log \frac{1}{x^{c}} \\
& <1+\frac{1}{\pi} \log \frac{1}{x^{c}} \\
& <1+\frac{d}{c} f_{\delta}\left(1-x^{d}\right) \log \frac{1}{x^{c}} \\
& =F\left(\frac{1}{2}-\delta, \frac{1}{2}+\delta ; 1 ; 1-x^{d}\right),
\end{aligned}
$$

which establishes (4.4). Inequalities (4.5) and (4.6) are immediate consequences of (2.11).

4.10. Conjectures. (1) For $c=2, d=3$ the best possible value of $\delta_{0}$ for which Theorem 4.3 is valid is $\delta_{0}=(\pi-2 \arcsin (2 / 3)) /(2 \pi) \approx 0.268$ (see [AVV3, p. 6]).

(2) Theorem 1.4 has a counterpart for the generalized hypergeometric function ${ }_{p} F_{q}\left(a_{1}, \ldots, a_{p} ; b_{1}, \ldots, b_{q} ; x\right)$ for the case $a_{i}>0, b_{j}>0, p=q+1$, when the sum is zero-balanced, i.e. when $\sum_{i=1}^{p} a_{i}=\sum_{j=1}^{q} b_{j}$. See also [B, p. 152].

\section{ACKNOWLEDGMENTS}

The research of the last-named author was supported by the Academy of Finland. The authors acknowledge useful conversations with K. Pearce.

\section{REFERENCES}

[AS] M. Abramowitz and I. A. Stegun, eds., Handbook of mathematical functions with formulas, graphs and mathematical tables, Dover, New York, 1965.

[A1] H. Alzer, Some gamma function inequalities, Math. Comp. 60 (1993), 337-346.

[AVV1] G. D. Anderson, M. K. Vamanamurthy, and M. Vuorinen, Functional inequalities for complete elliptic integrals and their ratios, SIAM J. Math. Anal. 21 (1990), 536-549.

[AVV2] __ Functional inequalities for hypergeometric functions and complete elliptic integrals, SIAM J. Math. Anal. 23 (1992), 512-524.

[AVV3] __ Hypergeometric functions and elliptic integrals, Current Topics in Analytic Function Theory (H. M. Srivastava and S. Owa, eds.), World Sci. Publ., Singapore and London, 1992, pp. 48-85. 
[AVV4] _ Inequalities for quasiconformal mappings in space, Pacific J. Math. 160 (1993), 1-18.

[Ask1] R. Askey, Ramanujan and hypergeometric and basic hypergeometric series, Ramanujan Internat. Symposium on Analysis (December 26-28, 1987) (N. K. Thakare, ed.), Pune, India.

[Ask2] _ Handbooks of special functions, A Century of Mathematics in America, Part III (P. Duren, ed.), Amer. Math. Soc., Providence, RI, 1989, pp. 369-391.

[Ba] L. Baker, C Mathematical Function Handbook, McGraw-Hill, New York, 1992.

[Be] B. C. Berndt, Ramanujan's notebooks, Part II, Springer-Verlag, Berlin, Heidelberg, and New York, 1989.

[BB1] J. M. Borwein and P. B. Borwein, Pi and the AGM, Wiley, New York, 1987.

[BB2] __ Inequalities for compound mean iterations with logarithmic asymptotes, J. Math. Anal. Appl. 177 (1993), 572-582.

[B] W. Bühring, Generalized hypergeometric functions at unit argument, Proc. Amer. Math. Soc. 114 (1992), 145-153.

[BF] P. F. Byrd and M. D. Friedman, Handbook of elliptic integrals for engineers and physicists, Grundlehren Math. Wiss., vol. 57, Springer-Verlag, Berlin, Göttingen and Heidelberg, 1954.

[C] B. C. Carlson, Special functions of applied mathematics, Academic Press, New York, 1977.

[Ev] R. J. Evans, Ramanujan's second notebook: Asymptotic expansions for hypergeometric series and related functions, Ramanujan Revisited: Proc. of the Centenary Conference (Univ. of Illinois at Urbana-Champaign) (G. E. Andrews, R. A. Askey, B. C. Berndt, R. G. Ramanathan, and R. A. Rankin, eds.), Academic Press, Boston, 1988, pp. 537-560.

[G] W. Gautschi, Some mean value inequalities for the gamma function, SIAM J. Math. Anal. 5 (1974), 282-292.

[LO] D. W. Lozier and F. W. J. Olver, Numerical evaluation of special functions, Proc. Sympos. Appl. Math. (to appear).

[Lu] Yu. L. Luke, Mathematical functions and their approximations, Academic Press, New York, 1975.

[Mi] D. S. Mitrinović, Analytic inequalities, Grundlehren Math. Wiss., Band 165, Springer-Verlag, Berlin, Göttingen, and Heidelberg, 1970.

[M] S. L. Moshier, Methods and programs for mathematical functions, Ellis Horwood, Chichester, 1989.

[PNB] W. F. Perger, M. Nardin, and A. Bhalla, A numerical evaluator for the generalized hypergeometric function, Comput. Phys. Comm. 77 (1993), 249-254.

[PT] B. A. Popov and G. S. Tesler, Computation of functions on electronic computers-Handbook, Naukova Dumka, Kiev, 1984. (Russian)

[PBM] A. P. Prudnikov, Yu. A. Brychkov, and O. Marichev, Integrals and series, Vol. 3: More special functions, transl. from the Russian by G. G. Gould, Gordon and Breach, New York, 1988.

[WW] E. T. Whittaker and G. N. Watson, $A$ course of modern analysis, 4th ed., Cambridge Univ. Press, London and New York, 1958.

Department of Mathematics, Michigan State University, East Lansing, Michigan

E-mail address: anderson@math.msu.edu

Department of Mathematics, Texas Tech University, Lubbock, TeXas 79409

E-mail address: narwbettacs1.ttu.edu

Department of Mathematics, Southwestern University, Georgetown, Texas 78626

E-mail address: richardk@ralph.txswk.edu

Department of Mathematics, University of Auckland, Auckland, New Zealand

E-mail address: vamanamu@mat.auckland.ac.nz

Department of Mathematics, University of Helsinki, FIN-00100 Helsinki, Finland

E-mail address: vuorinen®csc.fi 